\title{
Equivalence of Oblique and Frontal Illumination in Perspective Shape from Shading
}

\author{
Chi Kin Chow and Shiu Yin Yuen \\ Department of Electronic Engineering, City University of Hong Kong \\ Hong Kong, China \\ E-mail: \{chowchi, kelviny.ee\}@cityu.edu.hk
}

\begin{abstract}
In this paper, it is shown that any oblique illumination shape-from-shading problem under perspective projection for Lambertian reflection and a single distant light source can be converted to an equivalent frontal illumination problem by a simple nonlinear intensity transformation which is equivalent to a rectification in stereo vision. Remarkably, it involves no approximation of depth. The method is evaluated on perspective shape-from-shading involving wide range of oblique angles.
\end{abstract}

Index Terms - Shape from Shading, Perspective Projection, Oblique illumination, Rectification.

\section{INTRODUCTION}

Shape from shading is an inverse process to determine the depth map $D$ from its intensity image $I$ based on the fact that there is a relation between $D$ and $I$, i.e. $I=\mathrm{F}(D)$. Due to the difficultly of this problem, typically four assumptions are made in the past: (R1) Lambertian model; (R2) single light source placed at the infinity; (R3) orthographic projection and (R4) frontal illumination. For recent surveys, see Zhang et al. [1] and Durou et al [2].

Recently, several SfS algorithms relaxed the assumption of orthographic projection (R3) to a more realistic model (perspective projection). Yuen et al. [3] proposed perspective SfS with the Fast Marching method of [4]. This work is applied on images under frontal illumination. Another approach is reported by Courteille et al. [5], who considered the Eikonal equation obtained under frontal illumination and perspective projection. They used prior shape information to solve the equation.

The assumption of frontal illumination is too restrictive in realistic photographic conditions. As the light source direction is parallel to the viewpoint, either the light beam is occluded by the camera or the image of the light source is projected onto the image plane. Tankus et al. [6] suggest the use of an iterative solution of the perspective SfS problem under oblique illumination using the orthographic Fast Marching method of [4] as the initial solution. The experimental result under oblique illumination is not satisfactory. A detailed comparison of this method and the method of [3] (but under frontal illumination only) can be found in [3].
Prados and Faugeras [7] develop an iterative viscosity solution framework and a generic Hamiltonian that can handle orthographic as well as perspective projection under frontal or oblique illumination. Another interesting work is Prados and Faugeras [8], who show that the SFS problem has a unique solution and the unknown singular value point height problem is non-existent if the light source is placed at the center of projection and a distance attenuation term is included using the viscosity solution framework.

In this paper, we detach the problem of oblique illumination from that of perspective SfS, and present a solution to tackle the problem of oblique illumination. The proposed algorithm simplifies the problem from the oblique illumination case to the frontal illumination case by a coordinate system (CS) transformation, which provides a convenient method to solve a more realistic perspective SfS problem without approximation of depth.

Recently, Prados and Soatto [9] propose an extension of fast marching method that can deal with non-positive costs and orthographic as well as perspective projection under oblique illumination. One potential advantage of the fast marching formalism over the viscosity theory formalism is that the former is one-pass, whereas the latter is iterative (see [9] for preliminary numerical comparison).

This paper shows that the original, simpler fast marching with positive costs for perspective projection that applies for frontal illumination [3] is good enough for oblique illumination as well, albeit with rectification errors. Also, by our transformation, any new better numerical method (not necessarily fast marching or level sets, or viscosity) discovered in the future that works under frontal illumination can now also works under oblique illumination using our transformation (but subject to Lambertian and single far light source assumption).

This paper is organized as follows. Section II discusses an image transformation from the viewpoint CS to the light-source CS. Section III proposes a perspective SfS with oblique illumination algorithm based on the image coordinate transformation. In section IV, two sets of ex- 
periments illustrate the performance of the proposed algorithm. A conclusion is drawn in section V.

\section{IMAGE TRANSFORMATION}

Oblique light source SfS is the case that the light source direction is different from the viewing direction(s). The shading image in this case is:

$$
I(u, v)=\mathbf{l} \cdot \mathbf{n}=\left(l_{1}, \quad 0, \quad l_{3}\right) \cdot \frac{\left(-z_{x}, z_{y}, 1\right)}{\sqrt{z_{x}{ }^{2}+z_{y}{ }^{2}+1}}
$$

where $z_{x}$ and $z_{y}$ are the surface gradients along the $\mathrm{x}$ - and $\mathrm{y}$-axis for pixel $(u, v)$ respectively, and $\mathbf{I}$ is the unit vector of light source direction represented by two oblique angles $\mathbf{I}=[\alpha, \beta]$, i.e. $l_{1}=\cos \alpha \cos \beta, l_{2}=\cos \alpha \sin \beta$ and $l_{3}=$ $\sin \alpha$. To simplify the problem without loss of generality, $\beta$ is chosen as 0 , such that $l_{2}=0$ and the shading image is then given by

$$
I(u, v)=\left(l_{1}, \quad 0, \quad l_{3}\right) \cdot \mathbf{n}
$$

As Eq. (2) is not an Eikonal equation, some additional information is required to estimate a monotonic approximation to this term and an appropriate update rule.

In the case of orthographic projection, Kimmel and Sethian [4] adopted the suggestion of Lee and Rosenfeld [10] in which early SfS algorithms are improved by the light source coordinates. Kimmel and Sethian viewed the reflectance map as an 'almost' Eikonal equation. Together with the approximation of $z$ from its neighbor on the coordinate transformation, the equation can be solved. In this paper, the idea of coordinate transformation of [4],[10] is employed on the perspective model with oblique illumination. An image is transformed to the one viewed at the direction of light source (figure 1) by means of which the problem can be simplified as perspective SfS under frontal illumination.

Different from the orthographic projection case in [4], the coordinate transformation does not involve any approximation of $z$. So a more accurate result can be obtained. The details of the image transformation are discussed in the following:

Given that $I(u, v)$ and $D(u, v)$ are intensity and depth of the pixel $[u, v]$ respectively, the corresponding $3 \mathrm{D}$ coordinate $\mathbf{P}$ in viewpoint $\mathrm{CS}$ is:

$$
\left(\frac{u D(u, v)}{f}, \frac{v D(u, v)}{f}, \quad D(u, v)\right)
$$

On the other hand, the same point at the light-source CS is simply

$$
\left(D(u, v)\left(l_{1} \frac{u}{f}+l_{3}\right), \quad D(u, v) \frac{v}{f}, \quad D(u, v)\left(-l_{3} \frac{u}{f}+l_{1}\right)\right)
$$

where it is projected onto $\left[u^{\prime}, v^{\prime}\right]$ of the transformed image plane $\mathrm{H}^{\prime}$ under the perspective projection.

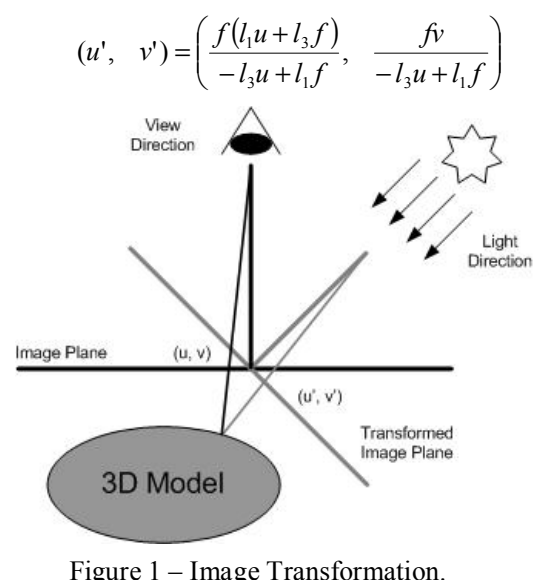

In general, the focal length $f$ may be different. Here we have used the same $f$ for simplicity.

Since the intensity is invariant to the viewing coordinate system under the Lambertian model (i.e. depends only on the surface normal and illumination direction), the intensity of the pixel $[u, v]$ at the image plane $\mathrm{H}$ is equivalent to the intensity of $\left[u^{\prime}, v^{\prime}\right]$ at the image plane $\mathrm{H}^{\prime}$, i.e. $I(u, v)=I^{\prime}\left(u^{\prime}, v^{\prime}\right)$, which is independent of $D$.

Note that technically, the above transformation is equivalent to a rectification step in stereo [11], in which the projected image is rectified to a virtual camera whose axis is in the illumination direction. However, the transformation is novel and has not been reported in the shape from shading literature to the best of our knowledge.

\section{THE PROPOSED ALGORITHM}

To tackle the problem of oblique illumination, we apply the proposed transformation to estimate the shading image $I_{f}$ (the image viewed at the illumination direction) as $I^{\prime}$. However, as the forward transformation from $(u, v)$ to $\left(u^{\prime}, v^{\prime}\right)$ does not uniformly cover all pixels at $I^{\prime}$, we apply a Sfs method at $\left(u^{\prime}, v^{\prime}\right)$ and calculate the one to one inverse projective transformation (homography) from ( $u$ ', $\left.v^{\prime}\right)$ to $(u, v)$ and then takes the intensity $I(u, v)$. This is exactly the pixel order scanning technique of texture mapping in computer graphics [12], which overcomes the inadequacy of the texture scanning. This re-sampling may cause aliasing errors [11],[12], which is commonly overcome in computer graphics by anti-aliasing. In this paper, a Sum of Gaussian (SOG) (or Gaussian Mixture Model $(\mathrm{GMM})$ ) surface interpolation is used as the anti-aliasing technique. This is generally considered a better surface interpolation technique than bi-cubic interpolation. Other anti-aliasing techniques may also be used.

Figure 2 shows an example of the proposed image transformation. Given a rendered image of the Mozart with oblique angles $\alpha=30^{\circ}$ and $\beta=0^{\circ}$ (figure 2a), the corresponding transformed image $I^{\prime}$ is shown at figure $2 \mathrm{~b}$. 
After recovering the depth map $D^{\prime}\left(u^{\prime}, v^{\prime}\right)$ of $I^{\prime}\left(u^{\prime}, v^{\prime}\right)$ by any frontal illumination perspective SfS algorithms, the depth map $D(u, v)$ of the given image $I(u, v)$ can be obtained by a geometric rotation. Similar to the image transformation, the GMM surface interpolation is performed to map from $D$ ' to $D$.

\section{EXPERIMENTS}

In this section, we first exemplify the proposed image transformation. Then we apply the proposed method to solve the perspective SfS problem under oblique illumination. Three standard synthetic models (see for example [3] for construction details): Sphere, Four Mountains and Mozart are used for the test. The total depths and the rendering parameters (focal lengths and offsets) of the models are listed at Table I

\section{A. Accuracy of the proposed illumination transforma- tion}

Suppose that a depth map $D$ is rendered to form the image $I$ with illumination direction $\mathbf{I}=[\alpha, 0]$, the proposed transformation aims at estimating the image $I_{f}$ directly from $I$ and $\alpha$. On the other hand, it can be exactly obtained from a sequence of projection and geometric transformations. Figure $2 \mathrm{~b}$ shows the desired $I_{f}$ of figure $2 \mathrm{a}$ and figure $2 \mathrm{c}$ is the $I_{f}$ estimated by the proposed transform.

In this experiment, the accuracy of the proposed illumination transformation is studied by comparing the similarity (root mean square (rms) intensity difference) between $I^{\prime}$ and $I_{f}$ of three models (The range of $I^{\prime}$ and $I_{f}$ are $[0,1]$.) Table II lists the similarities with $\alpha$ varied from $10^{\circ}$ to $70^{\circ}$. The scenario for Mozart at $70^{\circ}$ is not measured because at this large oblique angle, much is occluded and the image degenerates into several disconnected regions. As the oblique angle $\alpha$ increases, the distribution of the transformed pixels is sparser and the performance of the GMM surface interpolation is degraded slightly. Overall, the result shows that the errors due to the transformation are small even at large oblique angles.

\section{B. Perspective Fast Marching Method under Oblique Illumination}

This experiment shows the applicability of the proposed algorithm to extend the perspective SfS from frontal to oblique illumination. For each model, two oblique angle sets: $\left[30^{\circ}, 30^{\circ}\right]$ and $\left[40^{\circ}, 120^{\circ}\right]$ are used to render the images (figure 3). The perspective Fast Marching method of [3] is employed to recover the depth map of $I^{\prime}$. Other perspective SfS algorithms may also be used. The local minima are defined as the singular points and their depths are given [3],[6]. Meanwhile, the results are com- pared with those reconstructed by the method of Tankus et al. [6].

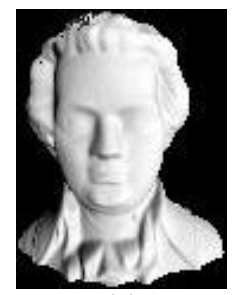

(a)

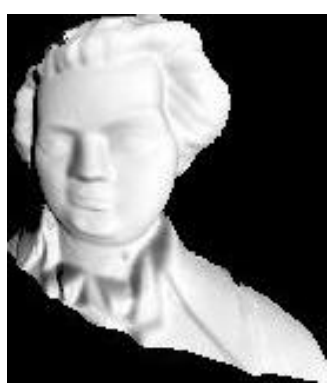

(b)

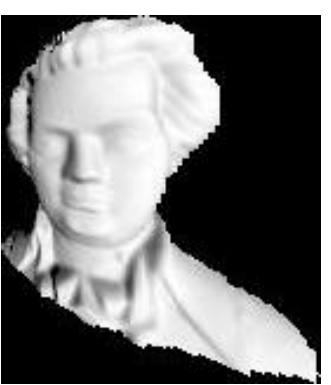

(c)
Figure 2 - Performance measurement of the proposed illumination transformation. (a) Input Mozart (b) Mozart viewed at the direction parallel to the light source (Ground truth) (c) Mozart viewed at the direction parallel to the light source (Estimated).

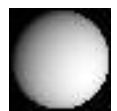

(a)

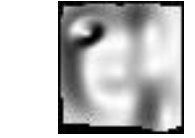

(c)

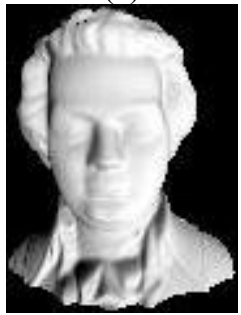

(e)

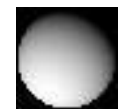

(b)

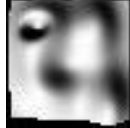

(d)

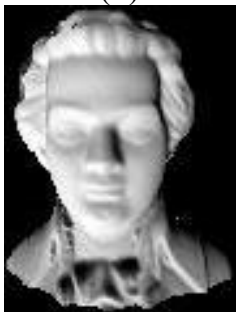

(f)
Figure 3 - The test images: (a) Sphere for $\mathbf{P}=\left[30^{\circ}, 30^{\circ}\right]$, (b) Sphere for $\mathbf{P}=\left[40^{\circ}, 120^{\circ}\right]$, (c) Four mountains for $\mathbf{P}=\left[30^{\circ}, 30^{\circ}\right]$, (d) Four mountains for $\mathbf{P}=\left[40^{\circ}, 120^{\circ}\right]$, (e) Mozart for $\mathbf{P}=\left[30^{\circ}, 30^{\circ}\right]$ and (f) Mozart for $\mathbf{P}=\left[40^{\circ}, 120^{\circ}\right]$.

TABLE I - TOTAL DEPTHS AND RENDERING PARAMETERS OF THE MODELS.

\begin{tabular}{|l||c|c|c|}
\hline & Total depth & Focal length & Offset \\
\hline \hline Sphere & 30 & 60 & 200 \\
\hline Four mountains & 42 & 70 & 200 \\
\hline Mozart & 95 & 65 & 200 \\
\hline
\end{tabular}


TABLE II - ERRORS OF THE PROPOSED ILLUMINATION TRANSFORMATION

\begin{tabular}{|c||c|c|c|}
\hline & Sphere & Four mountains & Mozart \\
\hline \hline$\left[10^{\circ}, 0^{\circ}\right]$ & 0.002886 & 0.005228 & 0.019778 \\
\hline$\left[30^{\circ}, 0^{\circ}\right]$ & 0.004718 & 0.00537 & 0.021992 \\
\hline$\left[50^{\circ}, 0^{\circ}\right]$ & 0.005848 & 0.01286 & 0.031079 \\
\hline$\left[70^{\circ}, 0^{\circ}\right]$ & 0.007915 & 0.016544 & - \\
\hline
\end{tabular}

TABLE III - SURFACE ERRORS AT $K=50 \%$ AND $75 \%$.

\begin{tabular}{|c||c|c|c|}
\hline $\begin{array}{c}\text { Oblique } \\
\text { angles }\end{array}$ & Sphere & $\begin{array}{c}\text { Four moun- } \\
\text { tains }\end{array}$ & Mozart \\
\hline \hline$\left[30^{\circ}, 30^{\circ}\right]$ & $1.182(6.67)$ & $1.682(21.52)$ & $1.037(9.09)$ \\
$1.791(8.11)$ & $2.698(23.47)$ & $2.012(12.818)$ \\
\hline$\left[40^{\circ}, 120^{\circ}\right]$ & $1.015(6.81)$ & $1.516(19.62)$ & $1.125(10.7)$ \\
& $1.359(8.31)$ & $2.347(22.36)$ & $2.381(13.21)$ \\
\hline
\end{tabular}

Figure 4 - 6 show that the proposed algorithm successfully recovers the depth maps of the perspective images with oblique illumination.

Quantitative comparison is a more tricky affair. Due to the discontinuities around the silhouettes of the tested models, it is expected that huge errors occur at that regions. Therefore, instead of rms depth error, the $k^{\text {th }}$ median absolute depth errors are used to measure the performance of the proposed algorithm. Table III lists the surface errors at $k=50 \%$ and $75 \%$. The values inside the brackets show the surface errors obtained by [6].

Seen from the table, the median errors of Mozart, Four mountains and Sphere are approximately equal as $\alpha$ is increased from $30^{\circ}$ to $40^{\circ}$. In the worst case, the ratio of median error to total depth is only $4.3 \%$. By comparing with [6], the proposed method estimates a significantly more accurate depth map under oblique illumination.

\section{CONCLUSIONS}

This paper proposes a robust algorithm to the problem of oblique illumination in perspective SfS. Instead of approximating the depths, the proposed algorithm estimates the intensities of sub-pixels by which a more accurate approximation is resulted. The algorithm transforms a shaded image from the viewpoint coordinate system (CS) to the illumination CS. Afterwards the resultant depth map can be obtained by applying any existing perspective SfS method on frontal illumination to the transformed image. The first experiment in section 4 shows the accurate estimation of the image viewed at the illumination direction obtained by the proposed transformation. The second experiment further illustrates the performance of the proposed transformation and the high adaptability to perspective SfS under oblique illumination. Previously, [3] has shown that it outperforms [6] under frontal illumination. Using the proposed transformation, it is shown that [3] outperforms [6] also in the case of oblique illumi- nation. Since the transformation is one step and simple, it involves a negligble additional computational overhead to [3].

The proposed transformation is equivalent to the well known rectification step in stereo vision, but its observation and application in this context in SfS is novel to the best of our knowledge.

Currently, the transformation proposed is only valid for the pure Lambertian model and a single light source at infinity. Future work is required to extend it to other models [7],[8].

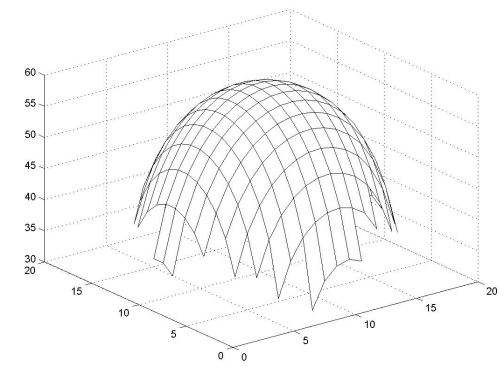

(a)

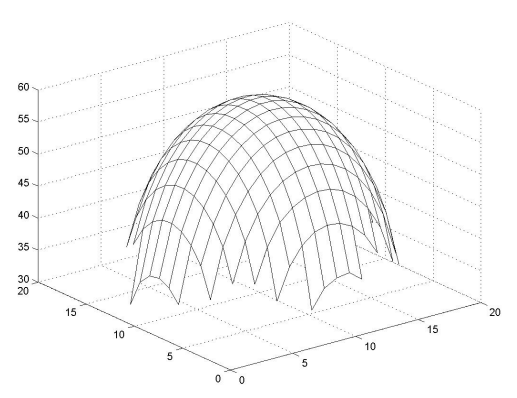

(b)

Figure 4 - reconstructed Sphere for the oblique angles (a) $\left[30^{\circ}, 30^{\circ}\right]$ and (b) $\left[40^{\circ}, 120^{\circ}\right]$.

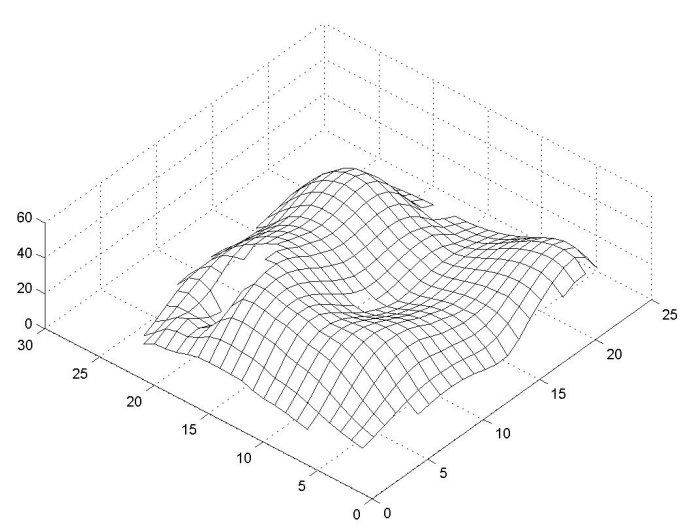

(a) 


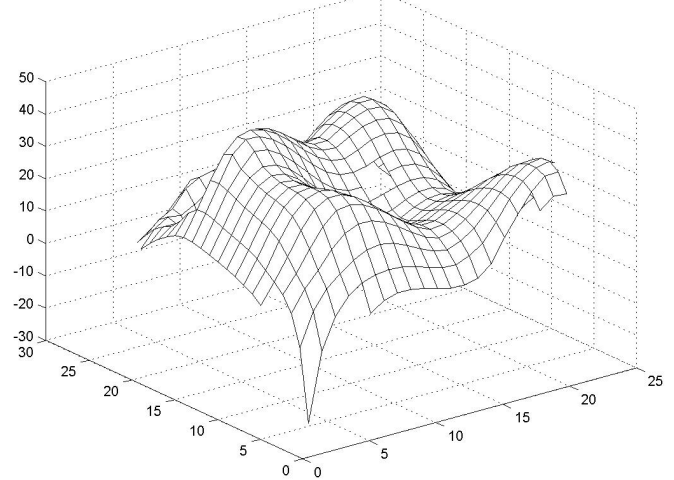

(b)

Figure 5 - The reconstructed Four Mountains for the oblique angles (a) $\left[30^{\circ}, 30^{\circ}\right]$ and (b) $\left[40^{\circ}, 120^{\circ}\right]$.

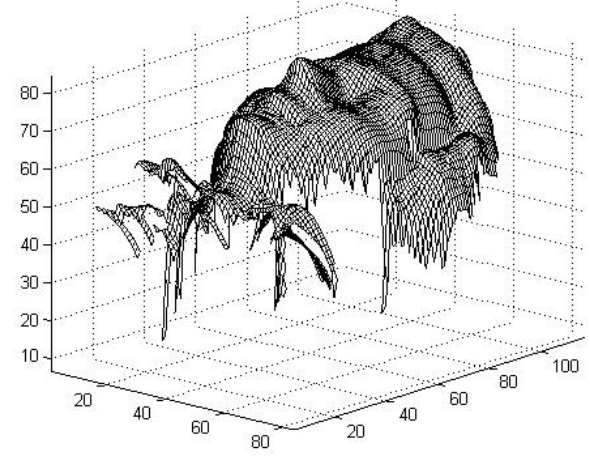

(a)

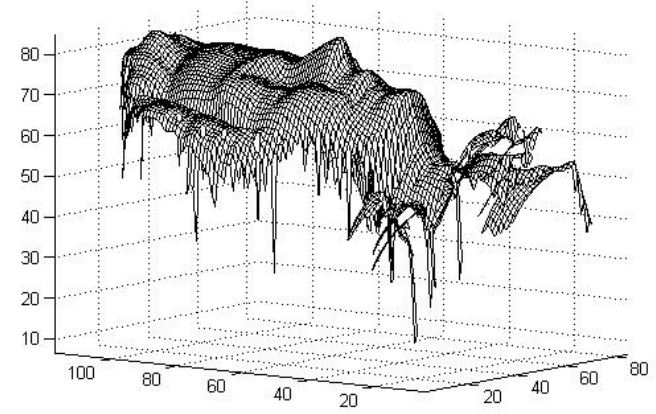

(b)

Figure 6 - The reconstructed Mozart for the oblique angles (a) $\left[30^{\circ}, 30^{\circ}\right]$ and (b) $\left[40^{\circ}, 120^{\circ}\right.$

\section{ACKNOWLEDGEMENTS}

The work described in this article was supported by a grant from CityU (7002128).

\section{REFERENCES}

[1] Zhang, R., Tsai, P.S., Cryer, J.E., Shah, M.: Shape from Shading: A Survey, IEEE T. PAMI, Vol. 21(8) (1999) 690-706.

[2] Durou, J-D., Falcone, M., Sagona, M.: A Survey of Numerical Methods for Shape from Shading, IRIT Technical Report 2004-2-R (2004).

[3] Yuen, S.Y., Tsui, Y.Y., Chow, C.K.: A Fast Marching Formulation of Perspective Shape from Shading under Frontal Illumination, PRL, Vol. 28 (2007) 806824.

[4] Kimmel, R., Sethian, J. A.: Optimal algorithm for shape from shading and path planning, JMIV, Vol. 14, No. 3, (2001) $237-244$.

[5] Courteille, F., Crouzil, A., Durou, J-D, Gurdjod, P.: Towards shape from shading under realistic photographic conditions, ICPR, Vol. 2. (2004) $277-280$.

[6] Tankus, A., Sochen, N., Yeshurun, Y.: Shape- fromshading Under Perspective Projection, IJCV, Vol. 63, No. 1. (2005) $21-43$.

[7] Prados, E., Faugeras, O.: A generic and provably convergent shape-from-shading method for orthographic and pinhole cameras, IJCV, Vol. 65(1-2) (2005) 97-125.

[8] Prados, E., Faugeras, O.: Shape From Shading: a well-posed problem? CVPR Vol. 2 (2005) 870-877.

[9] Prados, E., Soatto, S., Fast Marching Method for Generic Shape from Shading, LNCS Vol. 3752 (2005), 320-331.

[10]Lee, H., Rosenfeld, A.: Improved methods of estimating shape from shading using the light source coordinate system, Artificial Intelligence, Vol. 26, No. 1. (1985) $125-143$.

[11]E. Trucco, A. Verri: Introductory Techniques for 3-D Computer Vision, Prentice Hall (1998) or any standard computer vision text.

[12] Hearn, D., Baker, M.P.: Computer Graphics with OpenGL. $3^{\text {rd }}$ Ed. Prentice Hall (2004). 